\title{
A peripherally bolted torsional Langevin vibrator with large diameter
}

\author{
Yoshikazu Koike, Manabu Kihara, Jun Satonobu, Kentaro Nakamura, and \\ Sadayuki Ueha \\ Precision and Intelligence Laboratory, Tokyo Institute of Technology, \\ 4259, Nagatsuta, Midori-ku, Yokohama, 226 Japan
}

(Received 25 December 1996)

\begin{abstract}
To fabricate a bolt-clamped large diameter Langevin torsional vibrator as designed is usually difficult. A torsional vibrator tightened with an outside bolt is proposed in which the designed characteristics in the resonant vibration of the normal mode is achieved. A torsional vibrator 80 millimeters in diameter with a calculated resonant frequency of $28 \mathrm{kHz}$ has been fabricated. The resonant frequency of the torsional vibrator is $26.1 \mathrm{kHz}$, the quality factor is 890 and the maximum angular velocity is $4.1 \mathrm{rad} / \mathrm{s}$. It is confirmed that the vibration characteristics obtained with the externally-bolted transducer are improved compared with the torsional transducer tightened with an inside bolt.
\end{abstract}

Keywords: Bolt-clamped transducer, Torsional transducer, Externally-bolted transducer, Piezoelectric element, Ultrasonic transducer, Large diameter

PACS number: 43. 35. Yb, 43. 38. Fx

\section{INTRODUCTION}

In order to weld a large plastic surface using ultrasonic torsional vibration or to make a hybrid ultrasonic motor with a high output torque, it is necessary to have large magnitude vibrations in the transducer. Figure 1 shows a typical Bolt-clamped Langevin Torsional Transducer (BLTT). Two PZT elements polarized circumferentially are sandwiched by metal blocks. The polarized direction of one PZT element opposes the other. An inner stud bolt fastens the transducer together. An uneven of the clamping stress distribution occurs when the transducer is assembled. Since torsional vibration is propagated through shear forces, the clamping stress distribution of the interface between the components in a BLTT affects the vibration characteristics of the transducer. As a result, the distribution has serious influences on the properties of the torsional transducer, especially on its resonant frequencies and its maximum vibration amplitude of the resonant mode. In a bolt-clamped transducer, it should be noted that the characteristics of the torsional transducer could not attain the designed characteristics because of the unfavorable distribution of the clamping stress at the boundaries between the metal and the piezoelectric element. This effect appears with hybrid stators used in ultrasonic motors larger than $50 \mathrm{~mm}$ in diameter. The actual resonant frequency of the ultrasonic motors becomes lower than the designed frequency and the torsional vibration velocity is low. Ultrasonic motors less than $50 \mathrm{~mm}$ in diameter do not exhibit this behavior. ${ }^{1,2)}$ For a BLTT with $50 \mathrm{~mm}$ in diameter, K. Adachi achieves the designed properties when the structure of the BLTT is made into a hollow cylinder, the threads of the tightening bolt are adhered to the metal blocks by epoxy resin and contacting surfaces are finished by lapping with abrasive slurry. ${ }^{3)}$ Still these fabrication methods are not sufficient for larger diameter BLTT designs. On the contrary, we propose another fabrication method for a large diameter BLTT. The method employs a BLTT clamped together with an outside 


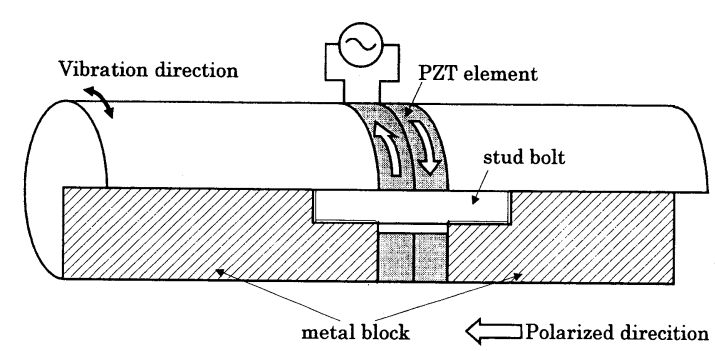

Fig. 1 The configuration of a typical Langevin torsional transducer.

bolt, differing from the conventional BLTT which uses an inner stud bolt. If the transducer is tightened by an outside bolt, the clamping stress area between the metal blocks and the piezoelectric element increases. Therefore, it is expected that the properties of the large diameter BLTT is improved and, hence, that the BLTT may achieve its designed properties. In this report, a hollow BLTT with 80 $\mathrm{mm}$ in diameter and tightened by an outside bolt is fabricated. The properties of the transducer approaches the designed properties in comparison with the internally-bolted BLTT.

\section{AN ESTIMATION OF THE VIBRATION PROPERTIES}

In this section, the vibration properties are estimated. At first, improvement of the vibration properties, especially the resonant frequency, of a large diameter BLTT has been simulated by using the finite element method (FEM). Figure 2 shows the simulation models. An internally-bolted transducers shown in Fig. 2(a) where it is assumed that an area exists along the outer circumference of the transducer where the clamped components do not touch. The externally-bolted transducer, however, has a small gap along the inner circumference of the transducer. In the simulation, the dimensions of the model are fixed at $80 \mathrm{~mm}$ in diameter and $56 \mathrm{~mm}$ in height. In Fig. 3, $R$ expresses the radius and $L$ expresses the length of the discontinuity. Varying the ratio of $L$ to $R$, the resonant frequency of the torsional mode is calculated by free vibration analysis. In Fig. 3, the calculation results are shown. The abscissa indicates the ratio $L / R$ and the ordinate indicates the resonant frequency of the first torsional mode normalized by the resonant frequency at $L / R=1$. It was found that the resonant frequency of an externally-bolted transducer drops

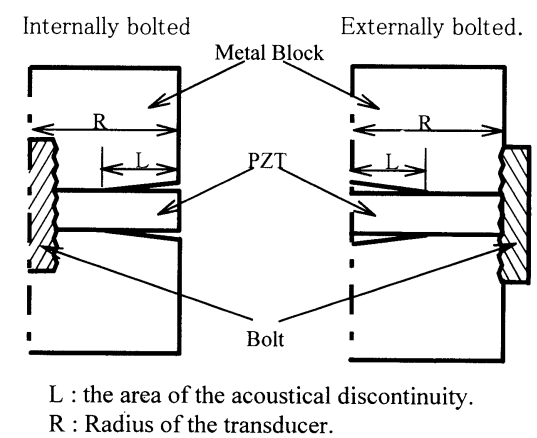

Fig. 2 The models of contact surface between PZT element and a metal block.

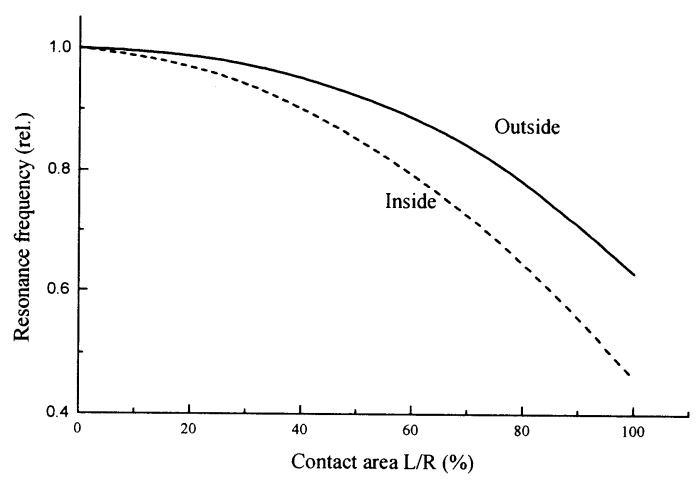

Fig. 3 The resonant frequency as a function of the ratio $L / R$.

less than the frequency of the internally-bolted transducer as the discontinuity area increases as shown in Fig. 3. Therefore, using an externally-bolted design can improve the properties of the transducer.

Secondly, the maximum angular vibration velocity is investigated. For simplicity, the transducer is assumed to be a uniform rod, that is, the fastening bolt and the PZT element are ignored. When the transducer is vibrated at the first torsional mode, the distribution of the angular vibration velocity, $\Omega$, along the axial direction $z$ is expressed by Eq. (1).

$$
\Omega=\Omega_{0} \sin k z,
$$

where $k$ is the wave number of the torsional mode. When the rigidity, the density and the diameter of the transducer are expressed by $G, \rho$ and $r_{0}$, respectively, the shear stress, $\tau$, of the torsional vibration mode is expressed by Eq. (2) using Eq. (1).

$$
\begin{aligned}
\tau & =\tau_{0} \cos k z \\
& =\tau_{0} \sqrt{\rho G} \Omega_{0} \cos k z .
\end{aligned}
$$


The shear stress between the PZT element and the metal block is almost equal to $\tau_{0}$. Using Eq. (1), $\Omega_{0}$ can be expressed by $\tau_{0}$ as indicated by

$$
\Omega_{0}=\frac{\tau_{0}}{r_{0} \sqrt{\rho G}} \text {. }
$$

With the distribution of the clamping stress, the static shear stress distribution of the boundaries may be estimated to be distributed. Using the friction coefficient, $\mu$, the static shear stress distributions, $\tau_{\mathrm{s}}(r)$, is expressed by the clamping stress distributions, $P_{\mathrm{s}}(r)$

$$
\tau_{\mathrm{s}}(r)=\mu P_{\mathrm{s}}(r)
$$

It is assumed that the angular velocity reaches a maximum when the vibratory shear stress given by Eq. (2) is equal to the maximum static shear stress given by Eq. (4). Compared with the internallybolted transducer, the contact area at the boundaries of the externally-bolted transducer increases. Therefore, the maximum clamping stress of the externally-bolted transducer is lower than that of the internally-bolted one when the tightening force is the same. As a result, it is predicted that the required tightening force in the externally-bolted transducer is larger in order to achieve the same angular vibration velocity of the internally-bolted transducer.

\section{CONSTRUCTION AND EXPERIMENT ON AN EXTERNALLY-BOLTED TOR- SIONAL VIBRATOR}

Figure 4 shows the construction of the prototype externally-bolted BLTT. Additionally, the picture of the parts of the transducers is shown in Fig. 5(a) and the picture of the assembled toransducer is shown in Fig. 5(b). The two PZT elements (FUJI

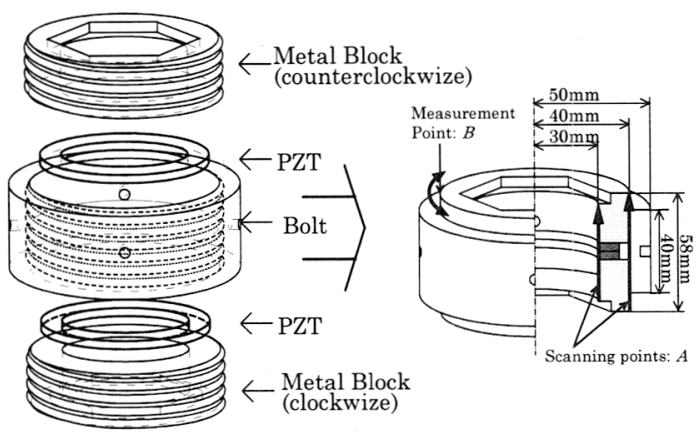

Fig. 4 A configuration of the externally-bolted torsional vibrator.
CERAMICS Co., Ltd.) with an outer diameter of 80 $\mathrm{mm}$, in an inner diameter of $60 \mathrm{~mm}$, and $4 \mathrm{~mm}$ in thick were used. Figure 6 shows the construction of the internally-bolted BLTT which corresponds to a conventional transducer. The metal blocks of both transducers are $25 \mathrm{~mm}$ in height and the total height of both transducers is $58 \mathrm{~mm}$. The metal blocks used in the externally-bolted transducer are hollow cylinders with an inner diameter of $60 \mathrm{~mm}$ and an outer diameter of $80 \mathrm{~mm}$. These dimensions match the PZT elements. The fastening bolt in the externally-bolted transducer has an outer diameter of $100 \mathrm{~mm}$, a height of $40 \mathrm{~mm}$. For the internally-bolted transducer, the tightening bolt has a diameter of $60 \mathrm{~mm}$ and a height of $50 \mathrm{~mm}$. Additionally, the center of the bolt has a hexagonal hole $30 \mathrm{~mm}$ across the widest diameter. The internally-bolted transducer is also a hollow cylinder. The upper part of both transducers shown in

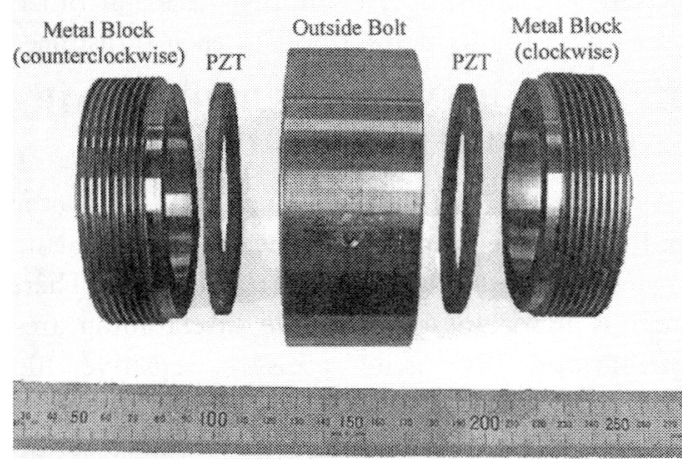

(a)

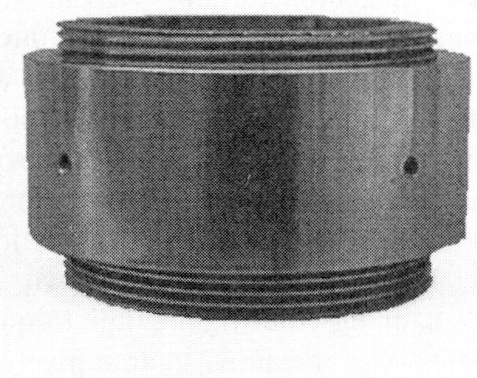

(b)

Fig. 5 The picture of the externally-bolted tortional transducer. (a) The parts of the transducer. (b) The assembled transducer. 


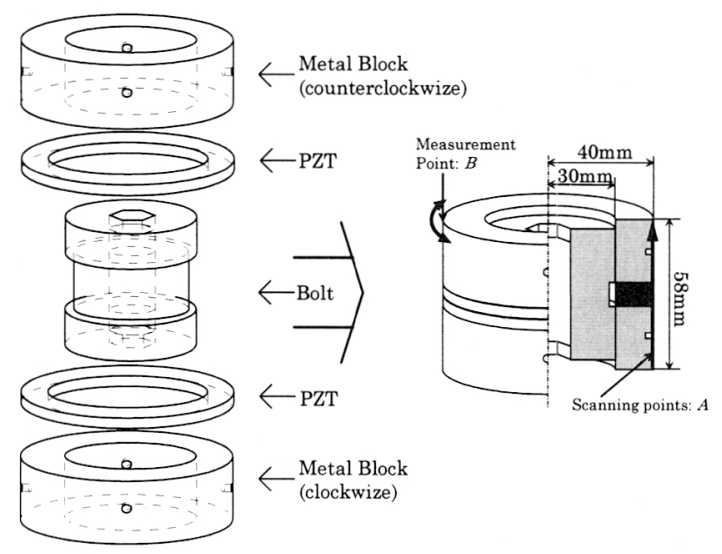

Fig. 6 A configuration of the internally-bolted torsional vibrator.

Figs. 4 and 6 has left-handed screw threads and the lower part has right-handed threads. Owing to these screw thread directions, the transducer can be screwed together simply clamping the metal blocks and turning the tightening bolt in each transducer.

\section{MEASUREMENT OF THE CLAMP- ING STRESS DISTRIBUTION}

As described in the introduction, the contact condition of the boundaries affects a great influence to the properties of the torsional transducer. Therefore, it is important to investigate the clamping stress distributions. By using pressure sensitive film (PRESCALE, FUJI PHOTO FILM Co., Ltd.), the clamping stress distribution of the contact surface between the PZT elements and the metal block was measured. The pressure sensitive film was put between the PZT element and the metal block. Figure 7 shows the results of the measurement at $6,000 \mathrm{kgf} \cdot \mathrm{cm}$ tightening torque. In Fig. 7(a), the pressure sensitive film was put in the internallybolted transducer and in Fig. 7(b), the film was put in the externally-bolted transducer. The clamping stress inside is higher than on the outside in Fig. $7(a)$, while the clamping stress along the outer circumference of the film is higher than that along the inner circumference of the film as shown in Fig. 7(b). By changing the tightening torque, the clamping stress was measured quantitatively. The absolute value of the clamping stress is measured by using special optical equipment (FPD-305, FUJI Photo Film Co., Ltd.). This optical equipment converts the coloring of the pressure film into an

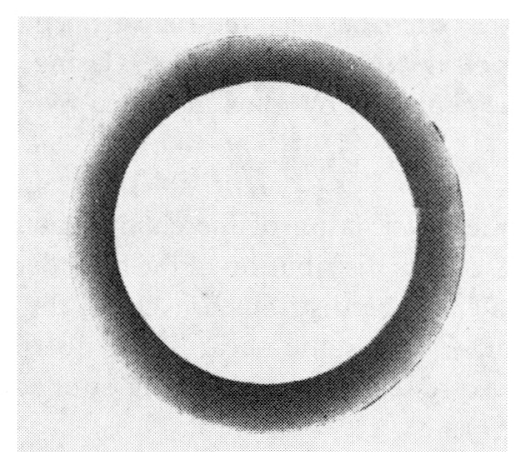

(a)

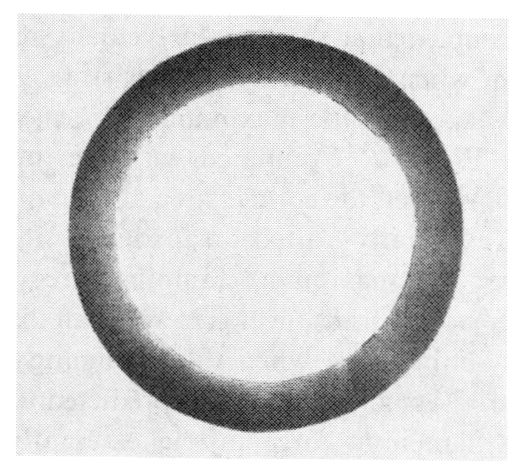

(b)

Fig. 7 The clamping stress distribution with a tightening torque $6,000 \mathrm{kgf} \cdot \mathrm{cm}$ for the (a) internally-bolted transducer, (b) externallybolted transducer.

absolute value of the normal stress. Figure 8(a) shows the clamping stress distributions of the internally-bolted transducer for several tightening torque. Figure $8(\mathrm{~b})$ shows analogous results for the externally-bolted transducer. The average of four points measured along the circumferential direction is plotted in each radial position. In the radial direction, the eight points are measured in intervals of $1 \mathrm{~mm}$. In both transducers, the clamping stress over the radial direction increases as the tightening torque increases. The clamping stress increases the most between $2,000 \mathrm{kgf} \cdot \mathrm{cm}$ and 3,000 $\mathrm{kgf} \cdot \mathrm{cm}$ for both transducers. As mentioned in the section 5, the electrical characteristics of the torsional transducer improves significantly between these values. The inner clamping stress is higher than the outer stress for the internally-bolted transducer, an opposite distribution to the stress in the externally-bolted transducer. The difference between the inner and the outer clamping stresses, 


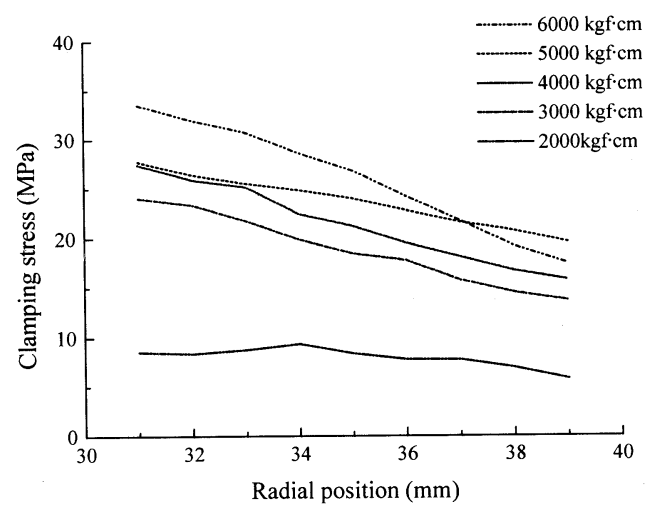

(a)

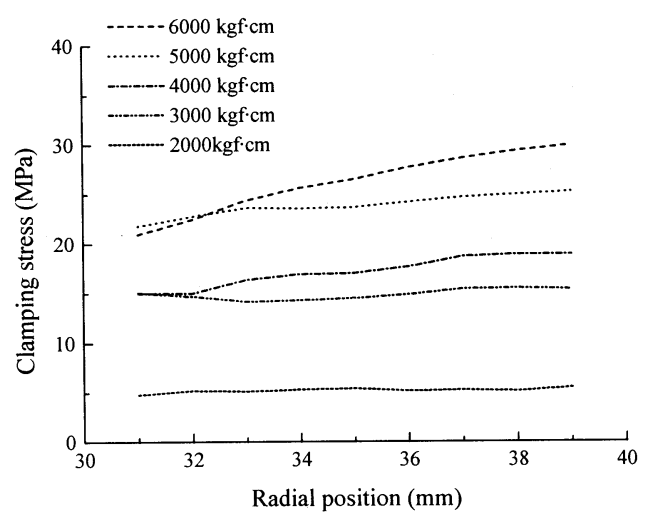

(b)

Fig. 8 The distribution of the clamping stress for the (a) internally-bolted transducer, (b) externally-bolted transducer.

however, becomes larger as the tightening torque increases. For the internally-bolted transducer, the outer clamping stress at $6,000 \mathrm{kgf} \cdot \mathrm{cm}$ is lower than at $5,000 \mathrm{kgf} \cdot \mathrm{cm}$. This drops in the clamping stress between $5,000 \mathrm{kgf} \cdot \mathrm{cm}$ and $6,000 \mathrm{kgf} \cdot \mathrm{cm}$ also appears for the externally-bolted transducer. Thus, merely tightening the transducer with a higher torque does not necessarily make the clamping stress over the entire contact surface larger. The clamping stress distribution in the externally-bolted transducer is more uniform than that in the internallybolted transducer for all tightening torque. Therefore, it can be expected that the properties of the externally-bolted transducer will be superior to the internally-bolted transducer.

\section{MEASUREMENTS OF THE ELECTRI- CAL PROPERTIES OF THE TRANSDUCERS}

The electrical properties of both prototype torsional transducers were measured. Figure 9 shows the variation of the resonant frequency and the $Q$ factor of the first torsional mode versus the tightening torque in both the externally-bolted and the internally-bolted transducers. An impedance analyzer (4192A, HP Co., Ltd.) was employed to measure the electrical properties of the transducers. The resonant frequencies of the first torsional mode shown in Fig. 9 are normalized by the calculated frequency for each type of transducer. The calculated resonant frequency for the internally-bolted transducer is $22 \mathrm{kHz}$ and $28 \mathrm{kHz}$ for the externallybolted transducer. The measured resonant frequencies in both transducers are close to the calculated frequencies as the tightening torque increases. The $Q$ factors also become higher. Judging from Fig. 8, the contact area increases as the transducer is fasten more strongly. This agrees with the estimated results shown in Fig. 3. When the tightening torque increases from $2,000 \mathrm{kgf} \cdot \mathrm{cm}$ to $3,000 \mathrm{kgf} \cdot$ cm, the $Q$ factor and the resonant frequency improves significantly in both transducers. As indicated in Fig. 8, this is the reason that the contact area between the PZT element and the metal block increases. For the externally-bolted transducer, the measured resonant frequency is approximately $10 \%$ lower than the calculated one at a tightening torque of $6,000 \mathrm{kgf} \cdot \mathrm{cm}$, while the measured frequency is about $20 \%$ lower than the calculated frequency in the internally-bolted transducer. The $Q$ factor at

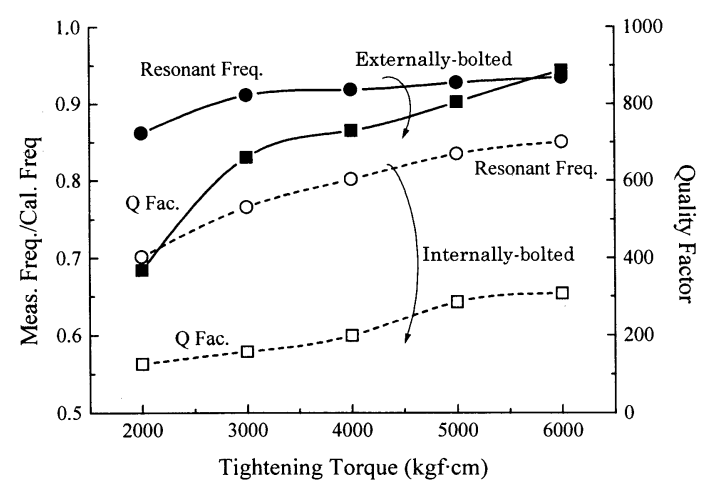

Fig. 9 Resonant frequencies and $Q$ factors as a function of the tightening torque. 


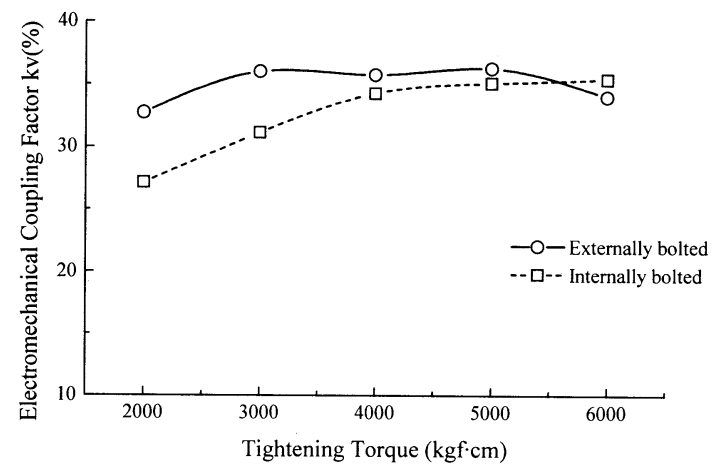

Fig. 10 Electromechanical coupling factors as a function of the tightening torque.

Table 1 Electrical characteristics of the transducers.

\begin{tabular}{lcc}
\hline & $\begin{array}{c}\text { Externally- } \\
\text { bolted }\end{array}$ & $\begin{array}{c}\text { Internally- } \\
\text { bolted }\end{array}$ \\
\hline $\begin{array}{c}\text { Calculation Resonant } \\
\text { frequency }(\mathrm{kHz})\end{array}$ & 28.0 & 22.0 \\
\hline $\begin{array}{c}\text { Measured Resonant } \\
\text { frequency (kHz) }\end{array}$ & 26.2 & 18.7 \\
\hline Quality Factor & 887 & 307 \\
\hline Motional admittance $(\mathrm{mS})$ & 182 & 71.8 \\
\hline $\begin{array}{l}\text { Electromechanical } \\
\text { coupling factor }(\%)\end{array}$ & 34.0 & 35.4 \\
\hline
\end{tabular}

890 in the externally-bolted transducer is approximately three times as high as in the internally-bolted transducer at 310. Therefore, it is confirmed that the torsional transducer fastened by an external bolt can greatly improve the vibration properties of the torsional mode. In Fig. 10, the electrical coupling factors are shown as a function of the tightening torque. Unlike the resonant frequencies and the $Q$ factors, the improvement of the externally-bolted fastening does not appear. This is because the tightening torque does not significantly affect the torsional resonant mode of the transducer. ${ }^{4)}$ In Table 1, the comparison of the electrical properties between the internally-bolted and the externallybolted transducer is shown at a tightening torque of $6,000 \mathrm{kgf} \cdot \mathrm{cm}$. In Fig. 11, the admittance loop for both transducers are shown. As shown in Fig. 11, the motional admittance is almost equal to the free admittance. When the transducer is fastened by the external bolt, the motional admittance reaches 182

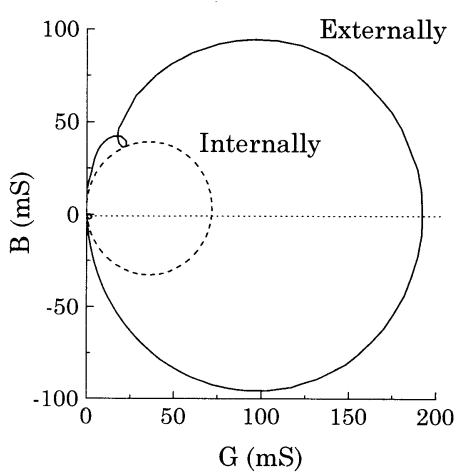

Fig. 11 The admittance loop with the tightening torque of $6,000 \mathrm{kgf} \cdot \mathrm{cm}$.

$\mathrm{mS}$. This is more than twice as much as the motional admittance of the internally-bolted transducer.

\section{MEASUREMENTS OF THE VIBRA- TION PROPERTIES OF THE TOR- SIONAL TRANSDUCERS}

The torsional vibration distributions were measured ; Fig. 12(a) shows the distribution of the first torsional resonant mode in the internally-bolted transducer and Fig. 12(b) is the distribution in the externally-bolted transducer. The angular vibration velocities were scanned along the axial direction $A$ as indicated in Figs. 4 and 6. In Fig. 12, the measured angular vibration velocities were normalized to the maximum value. In Fig. 12, the dotted line is the curve approximated by the sine function. As shown in Fig. 12(a) and (b), both transducers vibrate in the first torsional resonant mode. For the externally-bolted transducer, the angular vibration velocity at the outside bolt is lower than inside of the metal block. This is due to a vibration discontinuity between the metal block and the external bolt. The angular vibration velocity versus the motional current is plotted in Fig. 13. The measurement of the angular vibration velocity were made at the edge of the transducer indicated by $B$ in Figs. 4 and 6 . The motional current was calculated by dividing the input power by the applied voltage. The angular vibration velocity was measured with a Laser Doppler Vibrometer. For the internallybolted transducer, the angular vibration velocity was $4.3 \mathrm{rad}_{\mathrm{rms}} / \mathrm{s}$ with a motional current of $0.4 \mathrm{~A}_{\mathrm{rms}}$. For the externally-bolted transducer, the maximum angular vibration velocity obtained was $4.1 \mathrm{rad}_{\mathrm{rms}} / \mathrm{s}$ 


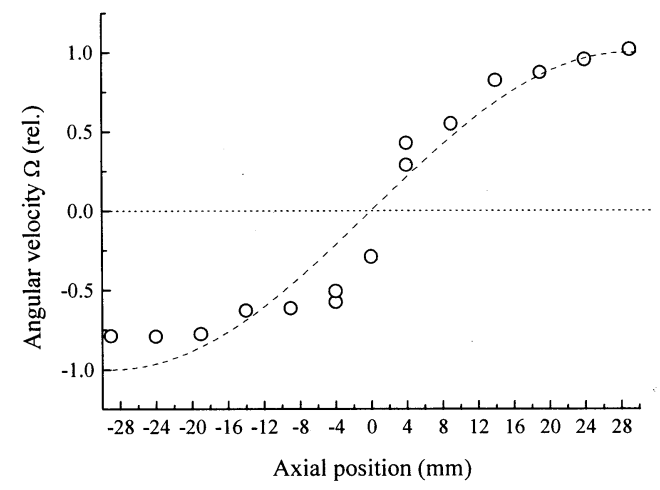

(a)

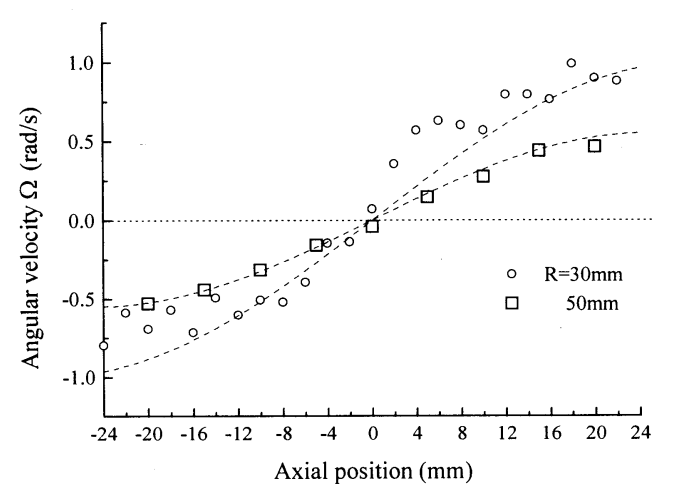

(b)

Fig. 12 The distribution of the angular velocity for the (a) internally-bolted transducer, (b) externally-bolted transducer.

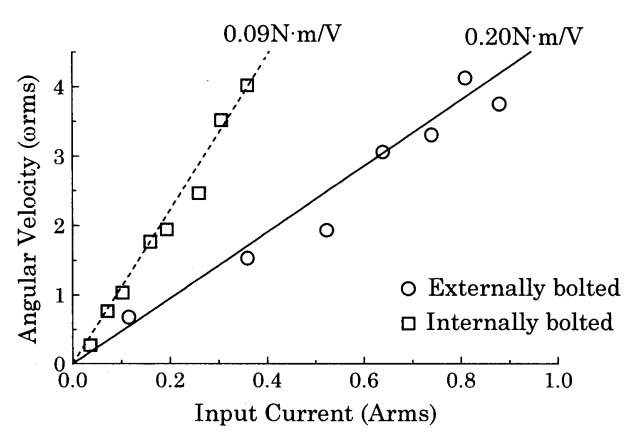

Fig. 13 The angular velocity as a function of the motional current.

at $0.8 \mathrm{~A}_{\text {rms }}$. At more than $0.8 \mathrm{~A}_{\text {rms }}$, however, the angular vibration velocity decreases. It is probable that slip was occurring between the PZT element and the metal in the transducers. For the maximum angular vibration velocity, the estimation
Table 2 The parameters used in the calculation.

\begin{tabular}{lc}
\hline The friction coefficient $\mu$ & 0.15 \\
\hline The inner radius $r_{1}$ & $30 \mathrm{~mm}$ \\
\hline The outer radius $r_{2}$ & $40 \mathrm{~mm}$ \\
\hline Density (S45C) $\rho$ & $7,800 \mathrm{kgf} / \mathrm{m}^{3}$ \\
\hline Rigidity (S45C) $G$ & $80.3 \mathrm{GPa}$ \\
\hline
\end{tabular}

described in Section 2 agrees with the result shown in Fig. 13, where the maximum velocity of the externally-bolted transducer is lower than that of the internally-bolted transducer. For the externallybolted transducer, the maximum clamping force is $30 \mathrm{~N} / \mathrm{m}^{2}$ at the outer radius. Using Eqs. (3) and (4) and the parameters indicated in Table 2, the maximum angular velocity, $\Omega_{0}$, predicted is 4.5 $\mathrm{rad}_{\mathrm{rms}} / \mathrm{s}$. This is almost equal to the measured maximum velocity shown in Fig. 13. For the internally-bolted transducer, the maximum angular velocity, $\Omega_{0}$, is $5.1 \mathrm{rad}_{\mathrm{rms}} / \mathrm{s}$ predicted by using Eqs. (3) and (4). In order to increase the maximum velocity in the externally-bolted transducer, it is necessary to clamp the transducer with a higher tightening force.

\section{CONCLUSIONS}

In order to successfully design and build a large diameter BLTT, we proposed a transducer fastened with an external bolt. The externally-bolted BLTT, $80 \mathrm{~mm}$ in diameter and $56 \mathrm{~mm}$ in height was made. It was compared with an internally-bolted transducer with the same dimensions. As a result, it was confirmed that the electrical properties of the externally-bolted transducer are much better than those in the internally-bolted transducer, however, the mechanical properties are almost the same. At a fastening torque of $6,000 \mathrm{kgf} \cdot \mathrm{cm}$, the characteristics obtained were a resonant frequency of $26.2 \mathrm{kHz}$ compared to a calculated frequency of $28 \mathrm{kHz}$, a $Q$ factor of 890 , an input admittance of $182 \mathrm{mS}$, an electrical coupling factor of $34 \%$ and a maximum angular vibration velocity of $4.1 \mathrm{rad}_{\mathrm{rms}} / \mathrm{s}$. The clamping stress distributions of both transducers were also measured. Using the measured distributions, it was confirmed that the maximum angular velocity is determined by the maximum clamping stress at the boundary surface between the PZT 
element and the metal block. In the future plan, the application of the externally-bolted transducer in a hybrid ultrasonic motor in order to enlarge output torque, may give an improved motor design.

\section{ACKNOWLEDGMENTS}

The authors thank the members of the machine shop in our laboratory, Mr. S. Okabe, Mr. N. Ouki, Mr. S. Wada and Mr. Y. Nagamine for their assistance in fabricating the experimental equipment. The authors also thanks Mr. James. R. Freind, a guest researcher from The University of MissouriRolla for helpful suggestions and observations.

\section{REFERENCES}

1) K. Nakamura and S. Ueha, "Performance of a hybrid transducer-type ultrasonic motor as a function of the size," Proc. IEEE 1994 Ultrason. Symp., 557-560 (1994).

2) J. Satonobu, N. Torii, K. Nakamura, and S. Ueha, "Construction of megatorque hybrid transducer type ultrasonic motor," Jpn. J. Appl. Phys. 35(9B), 50385041 (1996).

3) K. Adachi, Y. Konno, and S. Masaaki, "Development of bolt-clamped Langevin-type transducer with high mechanical quality factor for excitation of large torsional vibration," Jpn. J. Appl. Phys. 33(5B), 1182-1188 (1994).

4) M. Onoe, Y. Tomikawa, H. Jumonji, and Y. Mochizuki, Kotai Shindoron no Kiso (Ohmusha, Tokyo, 1982), Chap. 8, p. 175 (In Japanese). 